\title{
EDITORIAL
}

\section{Head and Neck Issues in Older Adults}

DOI:http://dx.doi.org/10.5770/cgj.17.149

The editorial board of the Canadian Geriatrics Journal is pleased to present our fall issue, which has a special focus on head and neck issues in older adults. Gomasani et al. present a study of patients in Nellore City examining the interaction between salivary changes and caries in older adults. Sharma et al. examine the compliance with radiotherapy for head and neck carcinoma in older adults in New Delhi. The relationship between perceived need for eye care services and available eye care services in a long-term care setting is described by Dr. Kergoat et al.

Hospital patients that are classified as an 'Alternate Level of Care' (ALC) are a growing part of the inpatient population. Dr. McClosky et al. examine the characteristics of ALC patients, specifically how the designation of ALC intersects with cognitive decline. Brothers et al. analyse in a European context how country of residence/country of birth predict performance-based cognitive and physical measures, congruent with previous work done on frailty measures. Role expectations for both specialists and family physicians in dementia care are presented using semi-structured qualitative interviews by Dr. Hum et al.

Have a great fall!

Dr. Kenneth M. Madden

Editor-in-Chief

Canadian Geriatrics Journal 